\title{
Shear and Stress in the Walls of a Paediatric Ventricular Assist Device
}

\author{
Vítor Augusto Andreghetto Bortolin ${ }^{1}$, Bernardo Luiz Harry Diniz Lemos ${ }^{1}$, Rodrigo de Lima Amaral ${ }^{1}$, \\ Marcelo Mazzeto ${ }^{2}$, Idágene Aparecida Cestari ${ }^{2}$, Júlio Romano Maneghini ${ }^{1}$ \\ ${ }^{1}$ Escola Politécnica da Universidade de São Paulo (EP USP) \\ Av. Prof. Mello Moraes 2231, São Paulo, Brazil \\ vitor.bortolin@usp.br; bernardolemos@usp.br; rodrigoamaral@usp.br; jmeneg@usp.br \\ ${ }^{2}$ Bioengineering Laboratory, Heart Institute and Medical Investigation Laboratory, Hospital das Clínicas, HCFMUSP, \\ Faculdade de Medicina, Universidade de São Paulo \\ Av. Dr. Enéas Carvalho de Aguiar 44, São Paulo, Brazil \\ marcelo.mazzetto@incor.usp.br; idagene.cestari@incor.usp.br
}

\begin{abstract}
Cardiac diseases are a major cause of death, unfortunately in serious conditions the only treatment is a transplant. However, the waiting line is long and most patients cannot wait until a suitable donor is found. This is a great issue specially for children due to their frail health. In this context, ventricular assist devices (VAD) improve patient's survival rates prolonging their lives. Notwithstanding, the construction of such devices is complex and even with the best available design blood degradation, through haemolyse and thrombocytes, is inevitable. However, a well design pump can minimize the damage but this is even more difficulty in children due to the large variation in body size. In this work, a paediatric assist device (PVAD) developed by InCor was studied to evaluate the shear rate and the viscous stress near the lateral wall. This was done using time resolved image velocimetry (TR-PIV). This is a critical region because is where the liquid can experience the highest stress or ever the greater stagnation. Therefore, most degradation occurs close to the walls. The results indicate a tendence towards low strain and stress unless for short periods in the peak of the diastole and systole in the vicinity of the walls.
\end{abstract}

Keywords: Ventricular Assist Device. Particle Image Velocimetry. Viscous Shear Stress. Erythrocytes. Thrombocytes.

\section{Introduction}

Cardiac failure in children is a serious health condition related to the presence of congenital heart disease and cardiomyopathies with an estimated incident in the population between 1-10 cases per 100.000 [1, 2]. In most cases, the treatment is a cardiac transplant. However, the waiting time for a suitable donor is long and the health condition of the patients is frail. Therefore, an intermediate treatment with a ventricular assist device (VAD) is needed to extend the life until the transplant. Besides, the treatment is an urgent need for anyone with in a severe cardiomyopathy, where the heart loses most of its pumping capacity. Still, the design and construction of these devices for paediatric use (PVAD) is a challenging task due to the wide range of corporeal sizes in children [3]. Beyond, the device must also have high reliability, low blood degradation or haemolysis, low thrombus and pannus formation, and even cost limitations for widespread use in large public health systems like the Brazilian SUS. Similar to other mechanical devices, the VADs are not comparable in function of a healthy heart, thus issues are present. The major concerning among pneumatic VADs is thrombus formation. When stagnation zones are present within the VAD's flow there is a chance of a clot to be formed. However, it cannot be stated that all stagnation zones will form clots. The relation between thrombus formation and stagnation zones in flow relies on viscous shear stresses (VSS) [3]. If the VSS, or its shear rate, is abnormally low there will be a huge chance of thrombus formation. In literature, shear rate values below $200 \mathrm{~s}^{-1}[4]$ or $500 \mathrm{~s}^{-1}[5,6]$ are considered dangerous to VAD operation. In contrast, high VSS or its shear rate $\left(>1000 \mathrm{~s}^{-1}\right)$ are associated to haemolysis [4]. Low VSS may occur due to internal geometry in stagnation regions while high shear, that lead to haemolysis, is more present near device walls.

In this context, the Bioengineering Laboratory of Heart Institute of InCor developed a pulsatile PVAD. This equipment is at the late development phase. At this stage, the device was tested to evaluate the flow pattern inside the device during a pump cycle. A series of experiments were performed using a time-resolved particle image velocimetry (TR-PIV) in a transparent device couple with a mock circulation loop (MCL) to study the flow behaviour. In a previous work [4], the temporal evolution of the velocity field in three distinct planes inside the PVAD were analysed. However, the viscous stress 
and shear acting over the fluid was not evaluated, specially near the walls. In these ventricular devices the low shear stresses near the device walls is the main responsible to initiate the coagulation cascade [7]. This is a cumulative process and for long periods of time can affect the patient's health. Thus, this work aims to evaluate the evolution of the shear and the viscous stress near the wall of a PVAD using the data collect by a time-resolved PIV.

\section{Methodology}

In this work, a non-intrusive measurement technique, time resolved particle image velocimetry (TR-PIV), was used to obtain the planar velocity field inside the paediatric device in a single cycle. This method allowed to study the flow with minimum interference because it need just optical access and tracing particles. However, the complex geometry of the PVAD was a major difficulty and guide the whole development of the experimental setup.

\subsection{Experimental Setup}

In the experiments, a transparent device received a custom-made wedge to reduce light scattering and reflex in the surface. This configuration allowed for the laser sheet to section the device in planes parallel to the centre of the valves. Figure 1a shows a draw of the top view of the wedge with the PVAD. The illumination system consisted of a diodepumped solid-state (DPSS) laser (Nd:YLF) with high repetition rate (up to $20 \mathrm{kHz}$ ) and double cavity (Litron Lasers Ltd, LDY304). The laser sheet produced had $5 \mathrm{~mm}$ in width and the energy of each pulse was $12.6 \mathrm{~mJ}$. The images were acquired with high speedy CMOS cameras (Vision Research, Phantom® Miro® R311) at a frequency of 3250Hz, at this rate the evolution of flow structures can be observed coherently, what characterizes a time-resolved system. In the acquisition, the single frame mode was used to allow for future multi-frame analysis increasing the dynamic range [5]. To compensate for the distortion inside the device a custom-made target was used to create a local correspondence between the image and the physical space. The final image resolution was $55.56 \mu \mathrm{m} / \mathrm{px}$. For trancing particles, it was used polyamide particles with mean diameter of $30 \mu \mathrm{m}$.

The pulsatile pump is actuated by a pneumatic system synchronized with the measurement apparatus. A flexible diaphragm without a net displaces the fluid in and out of the device. To control the flux in the pump two bi-leaflets unidirectional valves were used in the inlet and outlet. All valves were positioned along the horizontal axis of the pump, parallel to the diaphragm $\left(0^{\circ}\right.$ at Fig. 1a). A mixture of water, glycerine and ethanol was used to simulate blood with a similar density and viscosity. To simulate the pressure effect of a human body a mock circulation loop (MCL) was used (Fig. 1b). As stayed in [8], this system creates a similar hydrodynamic response that closes resemble the medical application. In all experiments the simulated cardiac frequency was kept constant at 70 BPM with systoles duration $30 \%$ of the total pump cycle. In every cycle 2785 images were acquired.
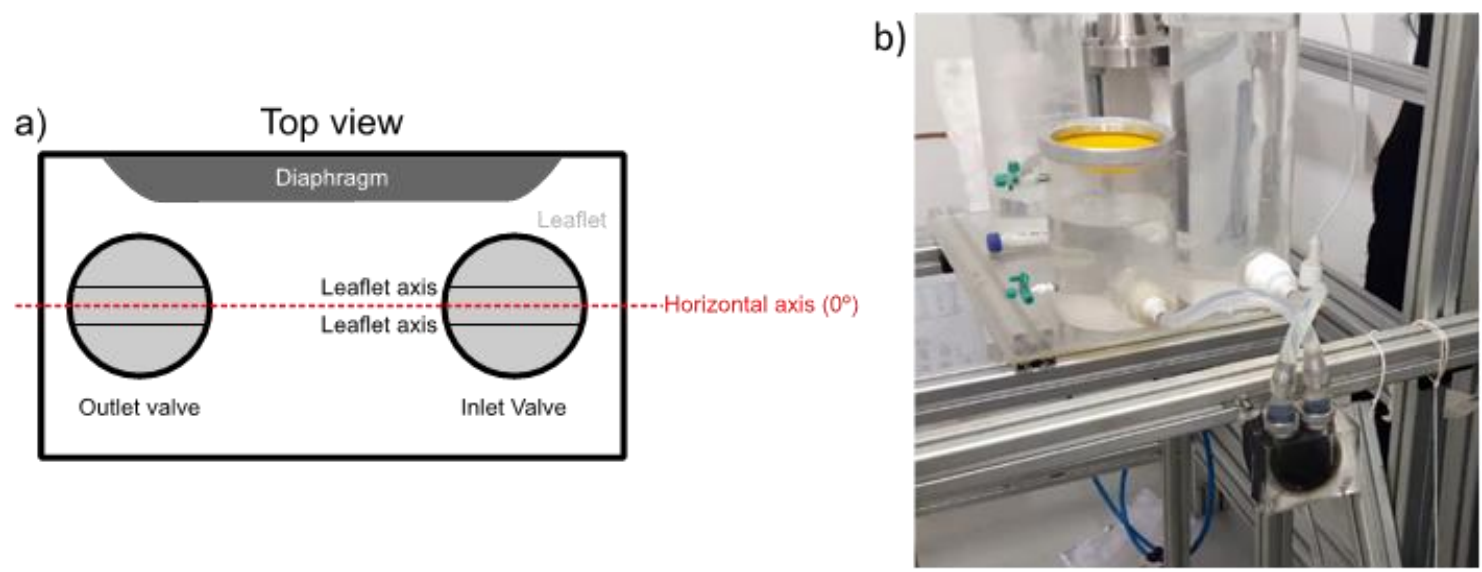

Fig. 1: Draw of the PVAD and a picture of the whole system.

\subsection{Wall definition and analysis}


The curvature inside the device and the transparent polymer causes optical distortions making difficulty to underline the correct position of the lateral walls in the acquired images. To solve this issue, a time average of the velocity field was done to determine the region with no movement. This, in association with the calibrated images, was used as a guide for manually selecting the wall region in the image. Two fixed walls were studied, one in the right, near the inlet valve, and another in the left side, below the outlet valve. In Figure 2, an example of the magnitude of the velocity field calculated by the TR-PIV is shown at the beginning of the diastole. The dark lines mark the geometric position of the walls and the dashed lines the limits of the valid data due to the shadows of the diaphragm and valves.

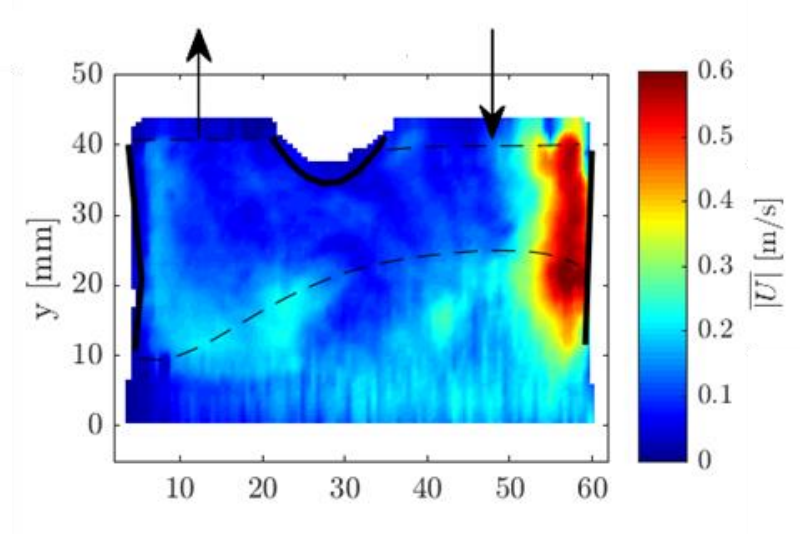

Fig. 2: Velocity magnitude field of PDAV at $20 \%$ of diastole

The analysis of the cycle was split between the systole and diastole phase determined by the electric signal that commands the air compressed valve feeding the diaphragm. Therefore, the whole cycle was split in interest intervals like the $20 \%-40 \%$ of the diastole period when a strong jet near the inlet wall is visible creating an elevated shear in the region. To calculate the instantaneous viscous shear stress (VSS) in this position the two-component field was used following equation 1. In this work, the average viscosity of the blood was used for this calculation. An important limitation of this calculation is the absence of the third component due to the planar measurements. However, the slim geometry of the chamber limits the out-plane motion near the lateral walls. In the case of the shear rate in the first vector after the wall that equation was greatly simplified to eq. 2 , where the value of the velocity in the perpendicular direction to the wall is divided by its distance to the wall. In this calculus, the maximum spatial resolution near the fixed geometry is a concern, due to high distortion and high velocity gradient this region is prone to. Therefore, it requires a fine grid to do not underestimate the stress. With a combination of a Gaussian, an RMS and an intensity cap filters over the undistorted and calibrated image the multi-pass standard cross correlation (SCC) algorithm achieved a final resolution of 15 pixels starting with a 32 pixels windows and $25 \%$ overlap. This is comparatively high resolution, especially when considering that the whole region had a signal-to-noise ratio (SNR) based on primary peak ratio (PPR) above 1.3.

$$
\begin{gathered}
V S S=\mu\left(\frac{\partial u}{\partial y}+\frac{\partial v}{\partial x}\right) \\
\text { shear rate } \text { wall }=\frac{v_{\perp \text { wall }}}{d_{\perp \text { wall }}}
\end{gathered}
$$

\section{Results}


Figure 3 shows the time series of the shear rate along the two walls inside of the PVAD through the whole beating cycle beginning with the diastole and finishing with systole. It is clear that at different moments there are gaps in the data. This is caused by the moving shadow of the diaphragm that decreases the SNR values to below the threshold of 1.3 thus only a small portion above $30 \mathrm{~mm}$ is always visible in both sets. Observing the shear for the right wall, there is a peak between approximately $200 \mathrm{~ms}$ and $400 \mathrm{~ms}$. Thus, that is the critical period for haemolysis in the cycle. The stagnation threshold, below $500 \mathrm{~s}^{-1}$, occurs in the end and in the beginning of the cycle in the portion of the wall closer to the valve. Meanwhile, the shear rate in the opposite wall is consistently lower than that of the right wall in the whole time series. It has two peaks, a bigger one in the end of the systole and another in the middle of the diastole, partially overlapping with the diastole peak. The secondary maximum in the left wall is associated with the recirculation movement formed inside the device after the diastole. The results in Figure 3, points out that the outlet region is more prone to the formation of thrombus or pannus insofar the wall near the inlet is more susceptible to haemolysis.
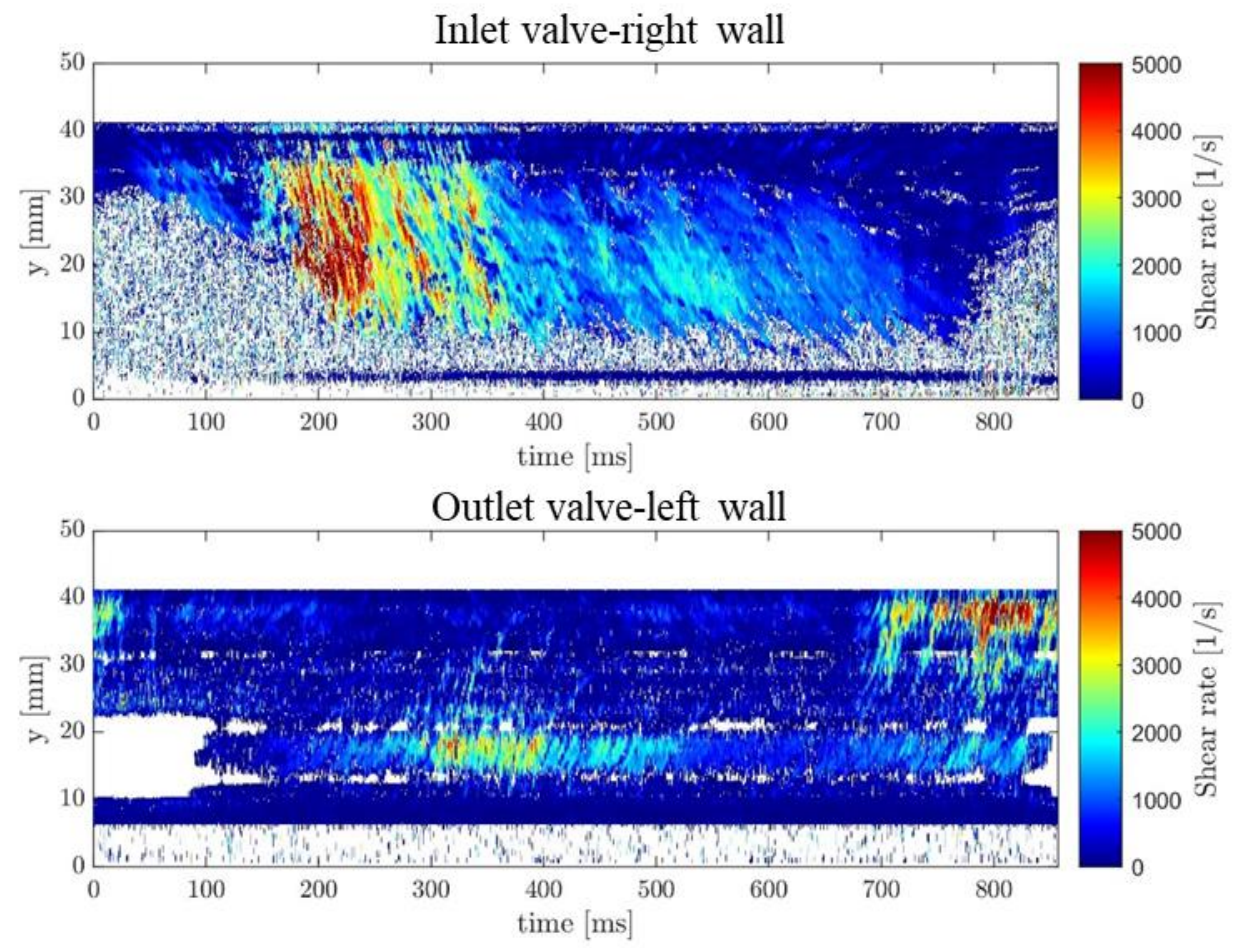

Fig. 3: Shear rate in the lateral walls of PVAD

Further analysing the data, the diastole phase was split in three intervals of approximately $120 \mathrm{~ms}$ corresponding to: $0 \%-20 \%, 20 \%-40 \%$ and $50 \%-70 \%$ of the diastole. Meanwhile the systole was portioned in another three periods of approximately $54 \mathrm{~ms}$ corresponding to: $0 \%-20 \%, 20 \%-40 \%$ and $80 \%-100 \%$ of the phase. Besides, a single observation point was selected at the y position of $35 \mathrm{~mm}$ (Fig. 4) where the walls are always visible, otherwise the viscous shear stress could not be calculated at all intervals. To plot the VSS in the time periods a perpendicular line was determined with $25 \mathrm{~mm}$ in length and the values of VSS were determined for all the points in this segment. Therefore, a twodimensional plot was created for each wall and for each time interval. 


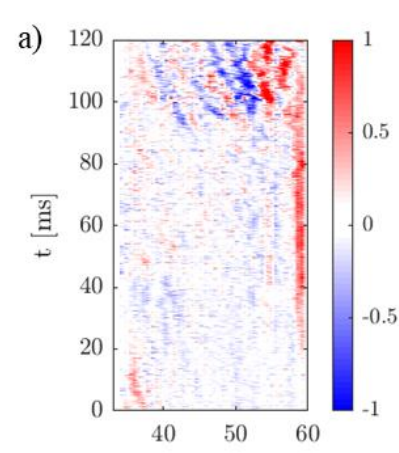

Inlet valve-right wall
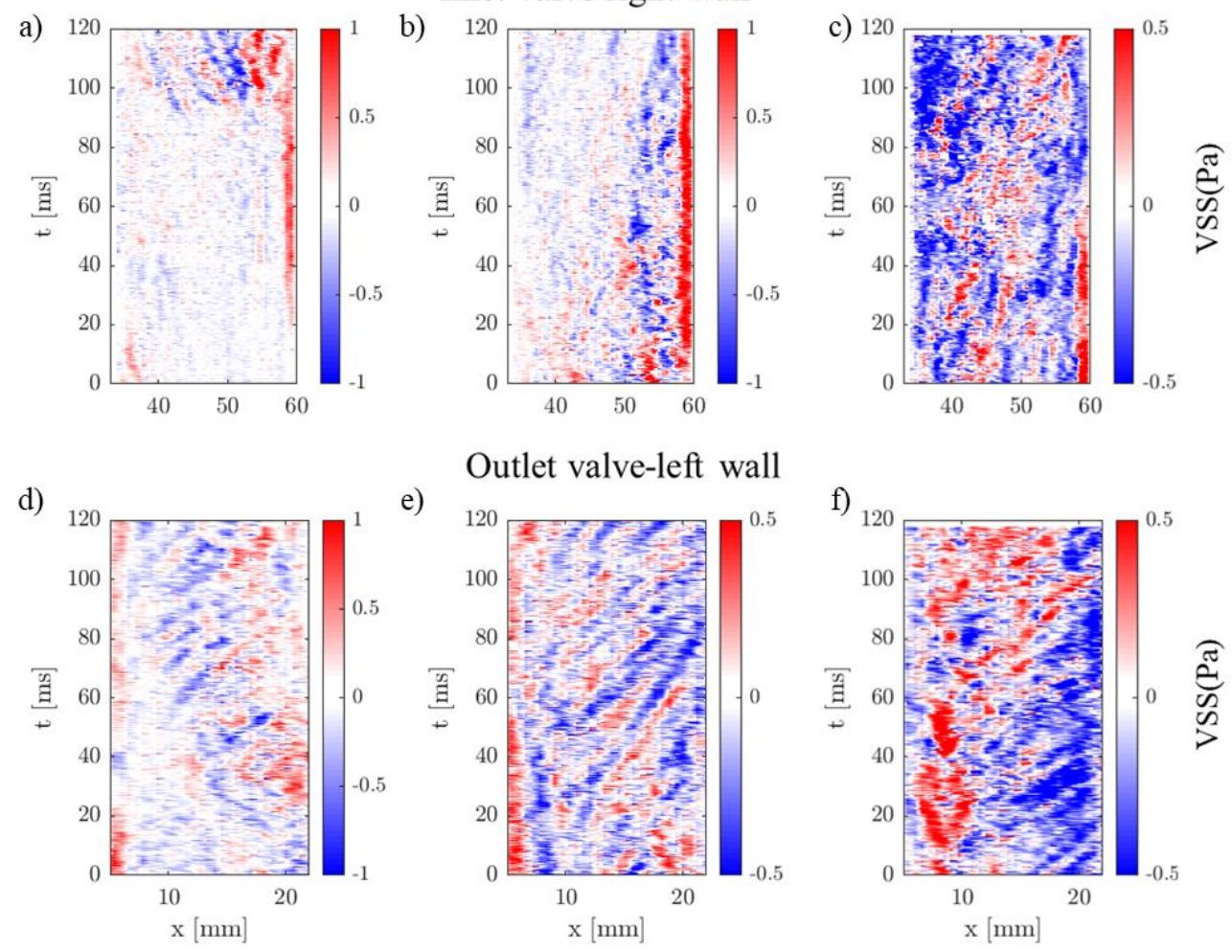

Fig. 4: Viscous shear stress over distance and time a) Right wall 0-20\% diastole; b) Right wall 20-40\% diastole; c) Right wall 50-70\% diastole; d) Left wall 0-20\% diastole; e) Left wall 20-40\% diastole; f) Left wall 50-70\% diastole.

In Figure $4 a, 4 b$ and $4 c$ is shown the right wall near the inlet valve. The $x$ coordinate is the same as in Figure 2, so the $60 \mathrm{~mm}$ mark correspond to the wall position. While in figure $4 \mathrm{~d}, 4 \mathrm{e}$ and $4 \mathrm{f}$ the $0 \mathrm{~mm}$ mark is the beginning of the wall. In the first $20 \%$ of the diastole there is a very low stress near the inlet wall specially when compared with the left region. This small delay of around $20 \mathrm{~ms}$ is related to the transition delay between the change in the solenoid valve, which allows compressed air to flow to pneumatic chamber, and the dynamical response of the flow. As the fluid fill the chamber a spike in the stress near the right wall is notice. Between $20 \%-40 \%$ of the diastole the VSS reaches a peak in the first $5 \mathrm{~mm}$ away from the wall and in the other side the distortion is limited and more uniformly distributed. In the last stage, the VSS is reduced in both observed lines and more spread out. The high stress near the wall track the peak inflow in the device beginning in the first $10 \%$ of the diastole until $60 \%$. Meanwhile, the stress at a distance, due to the recirculatory motion in the chamber, is small at the $0 \%$ but gradually increases as the chamber is filled and a vortical motion is formed. However, it is clear that the stress near the walls is the dominant effect because of its higher intensity. 


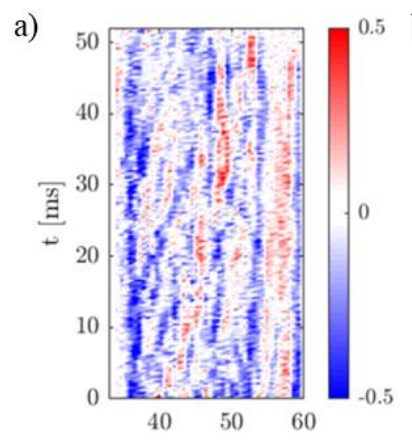

d)

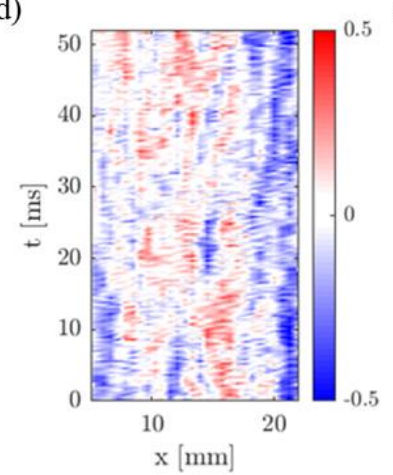

b)

Inlet valve-right wall

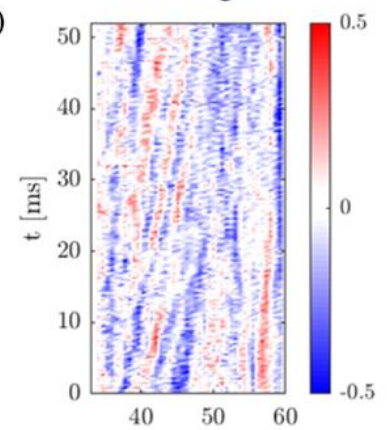

Outlet valve-left wall

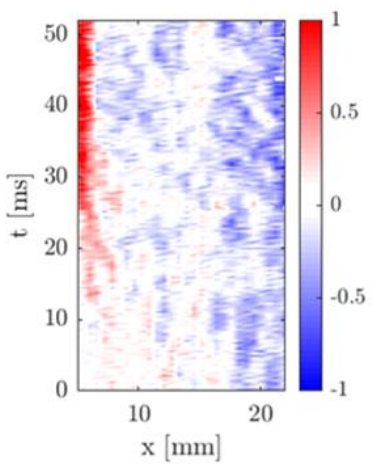

c)

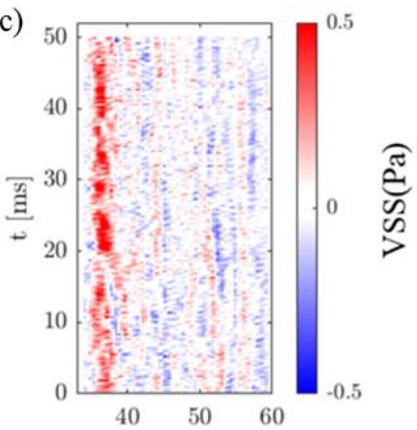

f)

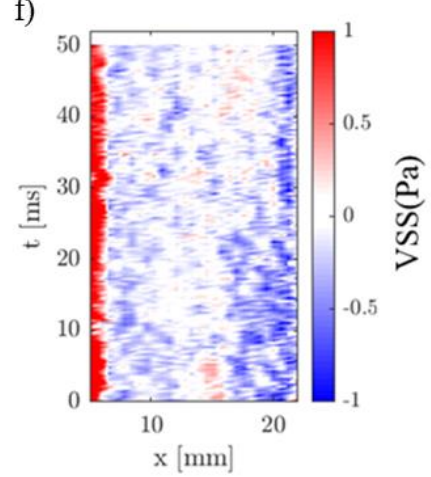

Fig. 5: Viscous shear stress over distance and time a) Right wall 0-20\% systole; b) Right wall 20-40\% systole; c) Right wall 80-100\% systole; d) Left wall 0-20\% systole; e) Left wall 20-40\% systole; f) Left wall 80-100\% systole.

Figure 5 show the same graphics disposition but for the three systole intervals. In the first $20 \%$ of the phase no clear difference in the stress profile is perceptible. There is a slight stasis period with the change in movement of the diaphragm. The inertia of the system results in a slow acceleration therefore, the shear is almost constant. However, between $20 \%-40 \%$ of the systole the line near the outlet exhibits an increasing strain close to the wall with values similar to the inlet in figure $3 \mathrm{~b}$. In the same period there is no substantial difference in the inlet area. Finally, in the end of the systole the VSS is continually high nearby the exit wall even as the cycle ends while the right wall suffers a reduction in stress level indicating a stagnation in that region. It is remarkable that the high stress near the left wall in figure $5 \mathrm{f}$ is still visible in the first $20 \mathrm{~ms}$ of image $4 \mathrm{~d}$. Thus, there is a slight overlap of the systole in the diastole and the same effect is not visible in the diastole. On the contrary, the inertial delay smooths the transition and reduces the overall stress.

This effect could be correlated with the shorter duration of the systole and the behaviour of the diaphragm and the air. When the diastole ends the diaphragm is contracted with low pressure inside the pneumatic chamber. When the solenoid valve switches, the compressed air will have to accelerate a large fluid mass that will exit the device. This process slows the systolic phase start. However, in the end of the systole the inertial effect pulls the diaphragm slightly more, prolonging the systole. 


\section{Conclusion}

In this work the viscous shear stress (VSS) and the shear rate in the walls of the PVAD, developed by the InCor, was analysed. The data indicates a concentration of shear rates during small intervals of the diastole and systole phases. Besides, the higher values occur during the peak of the emptying and filling and is far reduced during the recirculation specially in the end of the diastole and beginning of the systole. Besides that, the diaphragm movement coupled with the fluid inertia is the most probably explanation to the concentration of the shear in particular periods. Finally, the shear rates and VSS values were moderate in the majority of the cycle, therefore, the PVAD has a low probability to cause haemolysis but stagnation is a major concern with considerable regions with low shear or VSS during a prolonged period of time. The acquired data indicates that the left wall in the outlet region is more prone to stagnate while in the right wall near the intake there are short periods of intense shear and stress.

\section{Acknowledgements}

We gratefully acknowledge support of the RCGI - Research Centre for Gas Innovation, hosted by the University of São Paulo (USP) and sponsored by FAPESP - São Paulo Research Foundation (2014/50279-4) and Shell Brasil, and the strategic importance of the support given by ANP (Brazil's National Oil, Natural Gas and Biofuels Agency) through the R\&D levy regulation. This study was financed in part by the Coordenação de Aperfeiçoamento de Pessoal de Nível Superior - Brasil (CAPES) - Finance Code 001. This work was carried out with support from the Conselho Nacional de Desenvolvimento Científico e Tecnológico (CNPq), process 311191/2017-7. This work was supported by grant \#2012/50283-6, São Paulo Research Foundation (FAPESP). We thank the support of Financiadora de Estudos e Projetos (FINEP).

\section{References}

[1] J. W. Rossano, J. J. Kim, J. A. Decker, J. F. Price, F. Zafar, D. E. Graves, D. L. Morales, J. S. Heinle, B. Bozkurt, J. A. Towbin, S. W. Denfield, W. J. Dreyer and J. L. Jefferies, "Prevalence, Morbidity, and Mortality of Heart FailureeRelated Hospitalizations in Children in the United States: A Population-Based Study," Journal of Cardiac Failure, vol. v. 18, no. 6, pp. p. 459-470, 2012.

[2] B. B. Das, "Current State of Pediatric Heart Failure," Children, vol. 5, no. 7, p. 88, 2018.

[3] S. Deutsch, J. M. Tarbell, K. B. Manning, G. Rosenberg and A. A. Fontaine, "Experimental Fluid Mechanics of Pulsatile Artificial Blood Pumps," The Annual Review of Fluid Mechanics, vol. 38, pp. 65-86, 2006.

[4] P. Hochareon, K. B. Manning, A. A. Fontaine, J. M. Tarbell and S. Deutsch, "Correlation of In Vivo Clot Deposition With the Flow Characteristics in the $50 \mathrm{cc}$ Penn State Artificial Heart: A Preliminary Study," ASAIO Journal, vol. 50, no. 6, pp. 537-542, 2004.

[5] J. C. Nanna, M. A. Navitsky, S. R. Topper, S. Deutsch and K. B. Manning, "A Fluid Dynamics Study in a 50 cc Pulsatile Ventricular Assist Device: Influence of Heart Rate Variability," Journal of Biomechanical Engineering, vol. 133, pp. 101002-1 - 101002-10, 102011.

[6] M. A. Navitsky, S. Deutsch and K. B. Manning, "A Thrombus Susceptibility Comparison of Two Pulsatile Penn State 50 cc Left Ventricular Assist Device Designs,” Annals of Biomedical Engineering, vol. 41, no. 1, p. 4-16, 12013.

[7] A. Koliopoulou, S. H. McKellar, M. Rondina and C. H. Selzman, "Bleeding and thrombosis in chronic VAD therapy: focus on platelets," Current Opinion in Cardiology, vol. 31, no. 3, pp. 299-307, 52016.

[8] V. A. A. Bortolin, B. L. H. D. Lemos, R. d. L. Amaral, S. Bacht, M. Mazzeto, I. A. Cestari and J. R. Maneghini, "Temporal Flow Evolution on a Pediatric Ventricular Assist," in 7th International Conference of Fluid Flow, Heat and Mass Transfer, Niagara Falls, 2020.

[9] S. Burki and I. Adachi, "Pediatric ventricular assist devices: current challenges and future prospects," Vascular Health and Risk Management, vol. 13, pp. 177-185, 1552017.

[10] R. Hain and C. Kähler, "Fundamentals of multiframe particle image velocimetry (PIV)," Experiments in Fluids, vol. 42, no. 4, p. 575-587, 2007. 